\title{
The Relationship between Industrial Water Consumption and Economic Development_- the Strictest Water Resources Management System
}

\author{
Yue $\mathrm{Li}^{1, \mathrm{a}}$, Yanlong $\mathrm{Li}^{2, \mathrm{~b}}$ and Yang Yang ${ }^{3, \mathrm{c}}$
}

${ }^{1}$ College of Water Conservancy and Hydropower, Water Conservancy Project, Hebei University of Engineering, Handan City, Hebei Province, China

${ }^{2}$ Business School, Zhengzhou University, Econometric Analysis, Zhengzhou City, Henan Province, China

${ }^{3}$ Water Conservancy Engineering Department of Handan, Handan, Hebei Province, China

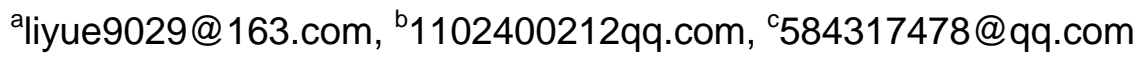

Keywords: Industrial water; Economic development; The strictest water resources management system; Spatial panel data model

\begin{abstract}
In this paper, it was based on the inter provincial panel data during the period from 2005 year to 2014 year. And it divided the 31 provinces into the East, the Middle and the West. Then this paper explored the Environment Kurtz Curve (EKC) between economic development and water use of each industrial area and discussed the optimal threshold. The results of this study show that: industrial water and economic development in the East, the Middle and the West regions was inverted "U" type; the corresponding per capita GDP of industrial water consumption per capita were 23644 RMB, 21549 RMB and 24433 RMB. The Strictest Water Resources Management System directly promoted the appearance of EKC inflection point in the East, the Middle and the West regions, as well as across the country. And it can achieve the "Double -Win" include industrial water consumption reduce and economic growth. The above results provide a theoretical and scientific support for the correct understanding of the changing law of water use and the effectively adjust the proportion of industrial water consumption and economic development.
\end{abstract}

\section{工业用水量与经济发展的关系一一最严格水资源 管理制度}

李月 ${ }^{1, a}$, 李彦龙 ${ }^{2, b}$, 杨阳 ${ }^{3, \mathrm{c}}$

1. 河北工程大学 水电学院、水利工程, 中国 河北 期䣋 056038

2. 郑州大学 商学院、经济计量分析, 中国 河南 郑州 450000

3. 期郸市水利工程处, 中国 河北 都䣋 056038

aliyue9029@163.com, ${ }^{b} 1102400212 @ q q . c o m,{ }^{c} 584317478 @ q q . c o m$

摘要: 该文基于 2005-2014 年省际面板数据, 将 31 省分为东、中、西部, 分别探索各地区工 业用水量与经济发展之间的环境库兹曲线 (EKC) 以及最优阀值。研究结果表明: 东、中、西部 地区工业用水与经济发展之间的关系曲线均呈倒 “U” 型; 东、中、西部人均工业用水量下降 点对应的人均 GDP 分别为 23644 元、21549 元和 24433 元; 最严格水资源管理制度直接促进 各地区 EKC 拐点的出现。这些都为正确把握用水变化规律、有效调控工业用水量与经济增长 提供了科学依据。

关键词：工业用水；经济发展；最严格水资源管理制度；空间面板数据模型 


\section{1. 引言}

水资源是兴盛工业的血脉, 是经济社会发展必不或缺的战略资源 $[1]$ 。然而水资源贯乏、水质 恶劣、水生态遭到破坏的现象层出不穷, 已经严重阻碍经济社会的快速发展。随着经济的高 速发展, 工业化水平也不断提高, 近十年工业用水虽只占总用水量的 $24 \%$ 左右, 但却是增长 速率最迅猛的淡水用户 [2]。由此可见, 工业用水量对缓解我国水资源压力、实现经济的可持 续发展起着至关重要的作用。因此, 工业用水量与经济发展的关系已然成为水资源领域的一 个重要研究问题。为解决水资源矛盾突出这一瓶颈, 实现工业用水高效利用, 亟需靠制度、 靠政策、靠改革从根本上撼动。最严格水资源管理制度就是从控制用水总量、提高用水效率、 控制水功能区限制纳污和实行水资源管理责任与考核制度四方面缓解我国水资源压力。

对于工业用水量相关研究国内外学者有不少讨论, 但对工业用水量与经济的研究却甚少。例 如贾绍风等基于水库涅茨曲线模型, 指出不论是发达国家还是发展中国家其工业用水量均不 会持续增长，当经济发展达到一定高度时工业用水量将会随之减少，两者间存在倒 “U” 型曲 线 [3]; Ridoutt、Hoekstra、Galan-de1-Castillo Elena 等均采用水足迹理论对水资源利用 与社会经济发展关系进行研究 [4-6]。本文旨在探索最严格水资源管理制度对工业用水量与经 济发展关系的影响，分析两者间是否存在环境库兹涅茨曲线，是否出现最优阀值。

\section{2. 指标选取与数据来源}

遵循指标选取的可应用性、科学性、严谨性等原则, 选取 2005-2014 年中国 31 省（市）的省 际面板面板, 如表 1 所示。

表 1 各变量汇总结果

\begin{tabular}{lll}
\hline 被解释变量 & 工业用水总量 & 人均工业用水量 $(\mathrm{IW})$ \\
\hline 解释变量 & 经济发展水平 & 人均 GDP ( 以 2004 年不变价进行调整) \\
\hline
\end{tabular}

数据来源于中国统计局以及各省市水资源公报等整理计算所得。众所周知, 我国经济存在明 显的发展不均衡状态, 若直接对全国进行分析, 必然会影响结果的严谨性, 故将 31 省分为东、 中、西 3 部分进行研究分析。具体划分情况见表 2 。

表 2 中国 31 省份分组情况

\begin{tabular}{lll}
\hline 区域 & 个数 & 省 (市)、自治区 \\
\hline 东部 & 11 & 北京、天津、河北、辽宁、上海、江苏、浙江、福建、山东、广东、海南 \\
中部 & 8 & $\begin{array}{l}\text { 山西、吉林、黑龙江、安徽、江西、河南、湖北、湖南 } \\
\text { 西部 }\end{array} 12 \begin{array}{l}\text { 内蒙古、广西、重庆、四川、贵州、云南、西藏、陕西、甘肃、青海、宁夏、 } \\
\text { 新疆 }\end{array}$ \\
\hline
\end{tabular}

\section{3. 模型建立}

本文在环境库兹涅茨曲线（EKC）经验 [7]的基础上采用空间面板数据模型对全国、东、中、 西部地区工业用水量进行分析。EKC 模型如 (1) 式所示:

$W=a_{0}+a_{1} Y+a_{2} Y^{2}+\varepsilon$

式中, W一人均工业用水量 (人均 IW) ; Y一人均 GDP; a0一常数项。

与传统的面板数据模型相比, 空间面板数据模型考虑到空间相关性的影响, 根据影响因子可 将面板数据模型分为空间误差模型 (SEM) 和空间滞后模型 (SLM)。根据自变量与随机扰动项 
之间相关性,空间面板数据模型也可分为空间固定效应和随机效应模型。通过 LR 和 Hausme [8] 检验，发现前者要优于后者，因此对于 SEM 和 SLM 的比较均基于空间固定效应模型。

SLM 是反映各种空间扩散产生的空间相关性, 主要集中体现在被解释变量的滞后项上, 其模 型形式如下:

$$
Y=\rho W Y+X \beta+\mu
$$

其中, $\rho$ 是空间自相关系数; $\mathrm{W}$ 是 $N \times N$ 维空间权重矩阵; $\mathrm{X}$ 是 $N \times K$ 的外生变量矩阵; $\beta$ 是 外生自变量的回归参数; $\mu$ 是满足正态独立分布的随机扰动项。

SEM 是反映空间相关性通过误差型得以体现的一种模型，其设定形式为:

$$
Y=X \beta+\varphi, \varphi=\delta W_{\varphi}+\mu
$$

其中, $\phi$ 为误差型; $W \phi$ 为空间自相关项; $\delta$ 是空间误差自相关系数; 剩余参数与 (2) 式 中的含义相同。

根据 Elhorst 改进的 LM 检验 [9] 比较 SEM 和 SLR 模型, 发现 SEM 模型更适合 2005-2014 年工 业用水量面板数据分析。综上所述，本文采用空间固定误差模型。

\section{4. 实证分析}

4.1. 全局空间自相关性分析

Moran’ I 检验 [10] 是 Pierce Moran 为了研究区域属性值得分布而提出的, 其计算公式如下:

$$
I=\frac{n}{S_{0}} \frac{\sum_{i=1}^{n} \sum_{j=1}^{n} W_{i j} z_{i} z_{j}}{\sum_{i=1}^{n} z_{i}^{2}}
$$

其中 $z i$ 是要素 $i$ 的属性与其平均值 $\left(x_{i}-\bar{x}\right)$ 的偏差; Wi j 是要素 $i$ 和 $j$ 之间的空间权重, 本 文选自经典的 $0-1$ 空间矩阵; $\mathrm{n}$ 等于要素总数; $\mathrm{S} 0$ 是所有空间权重的聚合: $\mathrm{S}_{0}=\sum_{i=1}^{n} \sum_{j=1}^{n} W_{i, j}$ 。 Moran' I 指数的绝对值在 0-1 之间，绝对值越接近 1 说明相关性越明显，反而越接近 0 说明 空间差异性越大。当莫兰指数大于 0 时，则表示空间呈正相关性，当莫兰指数小于 0 时，则 表示空间相关性为负相关，当莫兰指数等于 0 时，空间呈随机性。

本文采用 Matlab 软件计算十年间工业用水量的 Moran’ I 检验，检验结果如表 3 所示

表 3 2005-2014 年工业用水量的 Moran'। 检验

\begin{tabular}{llllllllllll}
\hline $\begin{array}{l}\text { 年 } \\
\text { 份 }\end{array}$ & 2005 & 2006 & 2007 & 2008 & 2009 & 2010 & 2011 & 2012 & 2013 & 2014 \\
\hline Mor & & & & & & & & & & & \\
an’ & 0.384 & 0.399 & 0.438 & 0.442 & 0.462 & 0.470 & 0.466 & 0.480 & 0.467 & 0.483 \\
I & $* * *$ & $* * *$ & $* * *$ & $* * *$ & $* * *$ & $* * *$ & $* * *$ & $* * *$ & $* * *$ & $* * *$ \\
\hline
\end{tabular}

注：＊＊＊水平显著性检验

从检验结果可以看出, 我国 2005-2014 年工业用水量 Moran’ I 值均为正, 对应的 P 值均小于 $1 \%$ 的临界值, 说明我国近 10 年工业用水量间存在空间正相关。另外由于 Moran' I 的值不断 增大，则说明 2005-2014 年间我工业用水量的空间相关性逐渐变强。

4.2. 回归分析 
由于上述两种模型存在空间相关性和异质性, 因此普通的 OLS 面板回归估计法已不适用 [11]。 本文根据 Anselin 的提出利用似然函数值对模型进行比较和检验, 即极大似然法 (ML) [12], 对模型进行估计。其结果如下表 4 所示。

表 4 各地区 SEM 估计结果

\begin{tabular}{lllll}
\hline & 全国地区 & 东部地区 & 中部地区 & 西部地区 \\
\hline \multirow{2}{*}{1 nGDP } & $2.502 * * *$ & $2.699 * * *$ & $7.743 * * *$ & $2.728 * *$ \\
& $(5.081)$ & $(4.615)$ & $(6.485)$ & $(2.012)$ \\
\multirow{2}{*}{$(1 \mathrm{nGDP}) 2$} & $-0.123 * * *$ & $-0.134 * * *$ & $-0.388 * * *$ & $-0.135 * *$ \\
& $(-5.030)$ & $(-4.838)$ & $(-6.405)$ & $(-1.932)$ \\
\hline
\end{tabular}

注： $* * * * * *$ 分别表示通过 $1 \% 、 5 \%$ 的显著性水平，括号里为回归系数 $\mathrm{T}$ 一统计量

表 5 各年份位于倒 “U” 型曲线右侧地区个数（单位：个）

\begin{tabular}{llllllllllll}
\hline & 2005 & 2006 & 2007 & 2008 & 2009 & 2010 & 2011 & 2012 & 2013 & 2014 & 共计 \\
\hline 东部 & 6 & 0 & 2 & 0 & 1 & 1 & 0 & 1 & 0 & 0 & 11 \\
中部 & 0 & 0 & 0 & 0 & 2 & 1 & 3 & 1 & 1 & 0 & 8 \\
西部 & 0 & 0 & 0 & 1 & 0 & 0 & 1 & 2 & 3 & 2 & 8 \\
全国 & 4 & 2 & 1 & 2 & 1 & 1 & 2 & 4 & 4 & 4 & 25 \\
\hline
\end{tabular}

由表 4 和表 5 可知:

（1）全国范围内 $1 n G D P$ 和 ( $1 n G D P ） 2$ 的系数均通过 $1 \%$ 的显著性检验, 说明在全国范围内的 工业用水量与经济发展之间呈倒 “ $U$ ” 型曲线。经计算, 该曲线的拐点值为 26127 元，而我国 的人均 GDP 在 2010 年就已经达到 30567 元, 说明我国现在正处在工业用水量库兹涅茨曲线的 右边, 即随着经济的发展, 工业用水量逐渐减少的有利局面。分省份比较可以发现北京、山 西等 25 个省的人均 GDP 均已超过拐点值, 即工业用水量随经济的增长而减少, 实现了工业用 水量减少和经济不断发展的 “双赢” 局面; 而安徽、广西、贵州、云南、西藏、甘肃这 6 省 的人均 GDP 还未超过 26127 元，即仍然处于工业用水随经济发展不断上升的 “两难” 困境。

(2) 从东、中、西三个地区分别来看, 东部地区 $1 n$ GDP 的系数为正, (1nGDP) 2 系数为负, 表明 EKC 的存在, 且为倒 “U” 型, 拐点值为 23644 元。在 2006 年东部地区的人均 GDP 就已 经超过 23644 元, 说明现阶段东部地区处于该曲线的右边。分省份来看, 在 2012 年前（包括 2012 年）东部地区 11 个省份均已达到工业用水量随经济增长而降低的 “双赢” 局面, 即均 已跨越拐点值。说明东部地区经济发展的迅猛以及工业化水平的先进。

（3）中部地区工业用水量与经济发展之间呈倒 “U” 型曲线，系数均通过显著性检验，同时 存在一个最优阀值, 即为 21549 元。中部地区处于该曲线的右侧。分省份来看, 中部地区中 8 个省全部在 2009 年以后出现高于拐点值 21549 元情况，有力的证明了最严格水资源管理制 度的实施对改善了工业用水与经济发展之间的关系起到了至关重要的作用。

(4) 西部地区中的 $1 n G D P$ 和 (1nGDP) 2 的系数均通过显著性检验, 即工业用水量与经济发展 之间呈倒 “U” 型曲线, 其拐点值为 24433 元。西部地区 2014 年人均 GDP 为 21881 元，还未 高于拐点值，仍处于曲线左侧，即工业用水量随经济发展而不断增加的局面。西部地区虽还 未跨越峰值，但在最严格管理制度促进了峰值的出现时间。分省开看，西部地区已有一半以 上在 2011 年左右实现了工业用水量与经济的可持续发展。

\section{5. 最严格水资源管理制度对工业用水量与经济发展关系的影响}

最严格水资源管理制度是中央为了改善我国的基本国情与水情而提出的。2005-2014 年经济 
社会迅速发展，2014 年 GDP 是 2005 年的 4 倍。但伴随着经济的快速发展资源消耗也随着而 来。为了解决问题, 亟需严格落实用水总量控制等 “四项制度”。以考核为抓手, 推动水资源 管理水平不断提升，实现水资源可持续利用保障经济社会持续健康发展的战略目标。

（1）在水资源管理方面。由过去的随性化、无序化、宽松化的管理模式转变为标准化、规范 化、严格化的管理。不仅从本质上推动了水资源利用方式的转变, 同时也促进了用水结构的 改善。东和中部在实施最严格水资源管理制度前其工业用水量基本都随着经济的增长而增长, 在实行该管理制度前后, 工业用水库兹涅茨曲线出现明显拐点, 随后发现其下降速度随着经 济的增长而增长, 可见最严格水资源管理制度的实施在很大程度上推动了拐点的出现。

（2）在 “四项制度” 实施方面。“四项制度” 的提出直接为最严格水资源管理制度提供了理 论指导, 不仅在用水总量上有所约束, 在用水效率方面也提出了多项要求, 这在一定程度上 对节约工业用水量创造了良好的条件，也为实现工业 EKC 曲线提高了强有力的保障。

（3）“三条红线” 的提出，进一步的支撑着最严格水资源管理制度的实施，不管是确立水资 源开发利用红线还是确立用水效率控制红线又或者是确立水功能区限制纳污红线, 都对工业 结构的优化、工业用水效率的提高、工业重复用水的力度的加强有着直接的影响, 间接影响 着经济社会的可持续发展。

\section{6. 结论}

本文基于空间面板模型以及库兹涅茨曲线对我国 31 省分区域分析工业用水量与经济发展之 间的关系，得到以下结论：

(1) 工业用水与经济发展的曲线关系直接说明工业用水量不会持续增长。当人均 GDP 达到一 定水平后工业用水将会呈下降趋势, 目前 31 个省中只有 19\%的省份还处于工业用水与经济发 展达到 “两难” 的局面, 其余省份均在实行最严格水资源管理制度前后跨越拐点值, 实现了 工业用水量与经济发展的 “双赢”。

(2) 不论是针对全国范围还是分地区讨论, 工业用水量与经济发展之间均呈现经典的 EKC 曲 线, 即倒 “U” 型曲线。说明在最严格水资源管理制度的实施下, 不久的将来工业用水量一定 会实现与经济发展共存的趋势。

(3) 最严格水资源管理制度的实施直接影响着工业用水量与经济发展之间的关系, 不仅促进 了两者间曲线转折点的提前出现，同时也影响着经济社会的可持续发展。

\section{7. 致谢}

基金项目：河北省科学技术研究与发展计划项目（12457201D-21）

\section{参考文献}

[1] 张兵兵,沈满洪. 工业用水与工业经济增长、产业结构变化的关系 [J]. 中国人口. 资源与环 境, 25(2),2015,9-14.

[2] 张陈俊,章恒全. 新环境库兹涅次曲线:工业用水与经济增长的关系 [J]. 中国人口. 资源与环 境, 05,2014, 116-123.

[3] 贾绍风,张士锋,杨红等. 工业用水与经济发展的关系一一用水库涅茨曲线 [J]. 自然资源学 报, 19(3),2004, 279-284.

[4] Ridoutt B G, Eady S J, Sellahewa J ,et al. Water footprint at the product brand level: case study and future challenges[J]. Journal of Cleaner Productiong, Vol. 17(2009) No. 13,p. 1228-1235.

[5] Hoekstra A Y, Chapagain A K. The water footprints of Morocco and the Netherlands: global water use as a result of domestic consumption of agricultural commodities [J]. Ecological Economics, Vol. 64(2007) No. 1,p. 143-151. 
[6] Galan-del-Castillo Elena, Velazquez Esther. From water to energy: the virtual water content and water footprint of biofuel consumption in Spain [J]. Energy Policy, Vol. 38(2010) No. 3, p. $1345-1352$.

[7] 杨万平,袁晓玲. 环境库兹涅茨曲线假说在中国的经验研究 [J]. 长江流域资源与环境, 08,2009, 704-710.

[8] Elhorst J P. Spatial panel date models [M]. In Handbook of applied analysis, M. M. Fischer and A. Getis, Berlin:Springer, 2010. 377-407.

[9] Anselin L, Bera A K, Florax R, et al. Simple Diagnostic Tests for Spatial Dependence [J]. Regional Science and Urban Economics,Vol. 26(1996)No. 1,p. 77 104.

[10]Anselin L, Getis A. Spatial Statistical Analysis and Geographic Infornation Systems [J].Annals of Regional Science, Vol. 26(1992) No. 1,p. 19-33.

[11]左丹. 基于空间面板模型的我国二氧化碳排放库兹涅茨曲线研究[D]. 西南财经大学

[12]Anselin, Luc, "Spatial Econometrics: Methods and Models," Dordrecht, Kluwer Academic Publishcrs, 1988, p.33-18.

\section{Acknowledgement}

This research was financially supported by the Hebei Province Science and Technology Research and Development Project (12457201D-21).And thanks the referees for their valuable comments and suggestions that have improved the paper immeasurably.

\section{References}

[1] Zhang Bingbing, Shen Manhong, "Relationship Between the Industrial Water Usage and the Industrial Economic Growth and the Industrial Structural Change," China Population, Resources and Environment, Vol. 25(2015) No.2, p.9-14.

[2] Zhang Chenjun, Zhang hengquan, "A New Environmental Kuznets Curve: Relationship between Industrial Water Use and Economic Growth," China Population, Resources and Environment, Vol. 24(2014) No. 5, p. 116-123.

[3] Jia Shaofeng, Zhang Shifeng, Yang Hong et al, "Relation of industrial water use and economic development water use Kuznet Curve," Journal of Natural Resources, Vol. 19(2004)No.3, p. 279-284.

[4] Ridoutt B G, Eady S J, Sellahewa J, et al, "Water footprint at the product brand level: case study and future challenges," Journal of Cleaner Productiong, Vol. 17(2009) No. 13, p. 1228-1235.

[5] Hoekstra A Y, Chapagain A K, "The water footprints of Morocco and the Netherlands: global water use as a result of domestic consumption of agricultural commodities," Ecological Economics, Vol. 64(2007) No. 1, p. 143-151.

[6] Galan-del-Castillo Elena, "Velazquez Esther. From water to energy: the virtual water content and water footprint of biofuel consumption in Spain," Energy Policy, Vol. 38(2010), No. 3, p. 1345-1352.

[7] Yuan Xiaoling, Yang Wanping, "Hypothesis of Environmental Kuznets Curve and its Test in China," Resources and Environment in the Yangtze Basin, Vol. 18(2009) No. 8, p. 704-710.

[8] Elhorst J P. "Spatial panel date models," in Handbook of applied analysis, M.M. Fischer and A.Getis, Berlin: Springer, 2010, p. 377-407. 
[9] Anselin L,Bera A K, Florax R,et al. Simple Diagnostic Tests for Spatial Dependence[J]. Regional Science and Urban Economics, Vol. 26(1996) No. 1, p. 77 104.

[10] Anselin L, GetisA, "Spatial Statistical Analysis and Geographic Infornation Systems," Annals of Regional Science, Vol. 26(1992) No. 1, p. 19-33.

[11]Zuo Dan, "An Analysis on China's carbon dioxide emissions Kuznets curve Based on spatial panel mode," Master, Statistics, Southwestern University of Finance and Economics, Sichuan, China, 2013.

[12] Anselin, Luc, "Spatial Econometrics: Methods and Models," Dordrecht, Kluwer Academic Publishcrs, 1988, p.33-18.

作者简介: 李月 (1990一), 女, 河北邯䣋, 硕士研究生, 水利工程, E-mai1：1iyue9029@163.com。 